Nonlinear Processes in Geophysics, 13, 41-51, 2006

SRef-ID: 1607-7946/npg/2006-13-41

European Geosciences Union

(C) 2006 Author(s). This work is licensed

under a Creative Commons License.

\title{
Evolution of localized vortices in the presence of stochastic perturbations
}

\author{
E. Bécu ${ }^{1}$ and V. Pavlov ${ }^{2}$ \\ ${ }^{1}$ Laboratoire de Mécanique de Lille, Boulevard Paul Langevin, 59655 Villeneuve d'Ascq, France \\ ${ }^{2}$ UFR de Mathématiques Pures et Appliquées, Université de Lille 1, 59655 Villeneuve d'Ascq, France
}

Received: 21 July 2005 - Revised: 11 November 2005 - Accepted: 18 November 2005 - Published: 10 March 2006

\begin{abstract}
We consider the evolution of a distribution of $N$ identical point vortices when stochastic perturbations in the Hamiltonian are present. It is shown that different initial configurations of vorticity with identical integral invariants may exist. Using the Runge-Kutta scheme of order 4, it is also demonstrated that different initial configurations with the same invariants may evolve without having any tendency to approach to a unique final, axially symmetric, distribution. In the presence of stochastic perturbations, if the initial distribution of vortices is not axially symmetric, vortices can be trapped in certain domains whose location is correlated with the configuration of the initial vortex distribution.
\end{abstract}

\section{Introduction}

There exist 2-D vortex systems, the evolution of which can be modeled by a great number of point vortices. One can suppose that a unique "gas" composed of $N \gg 1$ vortices of the same sign is a closed macroscopic system, it should evolve to a final state of statistical equilibrium, i.e. to an universal configuration.

In fact, two-dimensional turbulence evolution (even in the absence of dissipation and forcing) is governed by strongly nonlinear equations. On account of this, the field components interact intensively and have to quickly get mixed up. Therefore, one can assume that the system evolves to a state of statistical equilibrium that is axially symmetric.

Let us first discuss the physical meaning and validity of this largely used hypothesis.

For this purpose consider a system consist of a great number of point vortices. The angular velocity of each vortex $i$ is $\Omega_{i}=\dot{\theta}_{i}$ (cf Eq. 1) where $\theta_{i}$ is the angular vortex coordinate and $\dot{\theta}_{i}$ its temporal derivative). The magnitude of $\Omega_{i}$ can be estimated by $\bar{\Omega} \sim \Gamma / d^{2}, \Gamma$ being the total circulation and $d$ a characteristic domain diameter corresponding to the vortices

Correspondence to: E. Bécu

(emilie.becu@ed.univ-lille1.fr) location. Each vortex is influenced by all other surrounding vortices having a different angular velocity distributed about the mean angular velocity $\bar{\Omega}$ according to a certain law. That is why vortices, having for example the same values of $\theta_{i}$ at $t=0$ (i.e. $\theta_{1}=\theta_{2}=\ldots=\theta_{0}$ ) will disperse. Hence, the rapid process of vortices mixing with respect to angular position will occur.

Consider a function that depends on $\theta$ and $\Omega$ which describes the distribution of vortices and that at time $t=0 \mathrm{can}$ be written in the form $f\left(\theta_{0}, \Omega\right)$. Here, $\theta_{0}=\theta(t=0)$. Function $f\left(\theta_{0}, \Omega\right)$ can be decomposed in two parts:

$f\left(\theta_{0}, \Omega\right)=\bar{f}(\Omega)+f_{1}\left(\theta_{0}, \Omega\right)$,

where the averaged component,

$\bar{f}(\Omega)=(2 \pi)^{-1} \int_{0}^{2 \pi} d \theta_{0} f\left(\theta_{0}, \Omega\right)$,

does not depend on $\theta_{0}$. The function $f_{1}\left(\theta_{0}, \Omega_{i}\right)$ complies with the condition $\overline{f_{1}}\left(\theta_{0}, \Omega\right)=0$. Consequently, $f_{1}$ is a alternating-sign function with respect to the first argument. One can always find an interval $\Delta \theta_{12}=\theta_{01}-\theta_{02} \sim \pi$ where the sign of the function $f_{1}$ changes, i.e. $f_{1}\left(\theta_{01}, \Omega\right) \sim-f_{1}\left(\theta_{02}, \Omega\right)$. For $t>0$, $\theta_{i} \sim \theta_{0 i}+\Omega_{i} t$ and the distribution function becomes

$f(\theta-\Omega t, \Omega)=\bar{f}(\Omega)+f_{1}(\theta-\Omega t, \Omega)$.

Here, $\theta-\Omega t$ belongs to the interval $[0,2 \pi]$ (modulo $2 \pi$ ). For different values of $\Omega_{1}$ and $\Omega_{2}$, the terms which involves the time dependence become predominant in comparison with initial angles. Hence, the function $f_{1}$ changes sign when $\left(\Omega_{2}-\Omega_{1}\right) t \sim \pi$, i.e. for angular velocity variations $\Delta \Omega=\Omega_{2}-\Omega_{1} \sim \pi t^{-1}$. This simple estimation shows that $f_{1}=f-\bar{f}$ becomes an alternating-sign function with respect to the second argument as well. As $t \rightarrow \infty$, the interval between any $\Omega$ and $\Omega+\Delta \Omega$ becomes very small, i.e. $|\Delta \Omega| \ll|\bar{\Omega}|$, and the function $f_{1}$ becomes more and more oscillating with respect to the second argument $\Omega$. 
These arguments are of a general nature and can be applied to any variables ("phases") which change in a finite domain (Landau and Lifshitz, 1987; Landau and Lifchitz, 1979a, b).

In this way, the contribution of the alternating-sign function $f_{1}=f-\bar{f}$ is generally neglected. In fact, in real systems, the presence of a physical dissipation suppresses all processes on small time scales. The analogous mechanism (numerical dissipation) exists in numerical experiments when an artificial dissipation occurs at small scales. Finally, experimental processes retain a statistically indifferent intrinsic average caused by experimental conditions (cf for example experiments on study of quasi-final turbulence configuration Marteau et al., 1995; Danilov et al., 2002; Danilov and Gurari, 2000, and the references presented in these works).

In this context, several questions arise. If a real or an artificial dissipation is absent, what is the influence of nonvanishing fluctuations in the Hamiltonian of the system on possible scenarios of its evolution? Can different initial repartitions of vorticity be built which have the same global invariants (energy, enstrophy, moments, etc.)? If it is possible, do the systems, starting from different initial distributions of vorticity but having the same global invariants, evolve to a unique, universal, final state? Or do the systems evolve to different final states? Or even to no final state? Is it of vital importance on the processes of permanently existing small-scale end rapidly varying fluctuations? All these questions are motivated by the fact that systems with extremely weak dissipation are observed in nature.

\section{Different initial configurations with the same global invariants}

\subsection{Model}

We have to select a model complying with all conservation laws and which would be pertinent even on small space scales. In consequence, some of the traditional methods cannot be used and due to numerical difficulties at small scales. In fact, it is known that one of the difficulties in the description of turbulence is the expansion of the motion at small scales, to scales beyond those of viscous dissipation, when calculations come only from local mean-field at the scale of numerical resolution. The behavior of the turbulence, corresponding to explicit scales, is usually numerically modeled in a statistical sense; another way consists to introduce empirically different forms of turbulent viscosity which model energy transfer through intermediate scales, etc. However, it can be sometimes observed in two-dimensional turbulence that calculated magnitudes and phases of fluctuations of higher modes do not satisfy conservation laws. For this reason, we consider the model of point vortices, i.e. the model of elementary vortex structures for which the vorticity is strongly concentrated in small moving domains. This model is attractive for several reasons and has been largely used in many studies (cf Onsager, 1949; Miller et al., 1992;

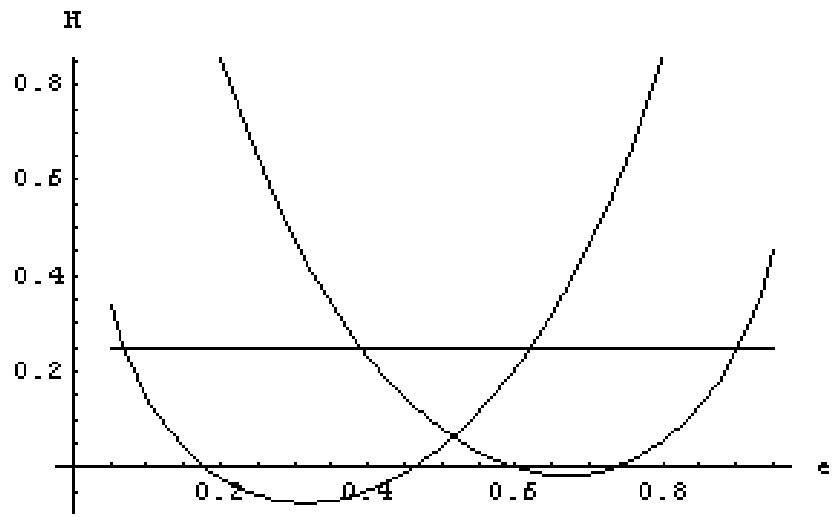

Fig. 1. The graphic representation of $e_{1}$ and $e_{2}$ corresponding to $H_{0}=0.25$ for $N=750$ and $K=260$.

Pointin and Lundgren, 1959; Sommeria et al., 1991; Brands et al., 1999; Pavlov et al., 2002 and references therein).

\subsection{Numerical simulations}

Consider an incompressible fluid containing $N \gg 1$ vortices with intensity $\gamma_{i}, i=1, \ldots, N$. The vorticity concentration is taken in the form $\Omega=\sum_{j} \gamma_{j} \delta\left(\boldsymbol{x}, \boldsymbol{x}_{j}\right)$ where $\delta^{(2)}\left(\boldsymbol{x}, \boldsymbol{x}^{\prime}\right)$ is Dirac's function. The equations of motion of the centers of the vortices are: $\gamma_{i} \partial_{t} x_{i}=\partial_{j} H, \quad \gamma_{i} \partial_{t} y_{i}=-\partial_{i} H$ which constitues a hamiltonian system. For the unbounded space, the Hamiltonian, i.e. kinetic energy of the fluid, expressed in terms of the canonical variables $x_{i}, y_{i}, H=\frac{1}{2} \int_{D} d \boldsymbol{x} v^{2}$, can be written in the form

$H=-(4 \pi)^{-1} \sum_{i, j ; i \neq j} \gamma_{i} \gamma_{j} \ln \left|\boldsymbol{x}_{i}-\boldsymbol{x}_{j}\right|$.

Here, $-(1 / 2 \pi) \ln \left|\boldsymbol{x}_{i}-\boldsymbol{x}_{j}\right|$ is a Green function which satisfies the equation $\Delta G\left(\boldsymbol{x}, \boldsymbol{x}^{\prime}\right)=\delta^{(2)}\left(\boldsymbol{x}, \boldsymbol{x}^{\prime}\right)$. The kinetic energy of the fluid can be written as $H=\frac{1}{2} \int_{D} d \boldsymbol{x} \psi \Omega$, with $d \boldsymbol{x}=d x d y, \Omega=-\Delta \psi$, and velocity components $v_{i}=\varepsilon_{i j} \partial_{j} \psi$, with $\varepsilon_{12}=-\varepsilon_{21}=1$ and $\varepsilon_{11}=\varepsilon_{22}=0$.

For the following numerical simulations performed in the present work, it is convenient to use dimensionless variables. The transformation is accomplished by $x_{i} \rightarrow R x_{i}, \quad y_{i} \rightarrow R y_{i}, \quad t \rightarrow \tau t, \quad$ where $\tau=2 \pi R^{2} \Gamma^{-1}$. Note that the vortices have a identical intensity $\gamma_{i}=\Gamma / N$. Let $\left(x_{i}, y_{i}\right) \rightarrow\left(\theta_{i}, J_{i}\right)$, with $x_{i}=\sqrt{2 J_{i}} \sin \theta_{i}$ and $y_{i}=\sqrt{2 J_{i}} \cos \theta_{i}$. The variables $\left(\theta_{i}, J_{i}\right)$, where $J_{i} \equiv r_{i}{ }^{2} / 2, \quad r_{i}=\left|\boldsymbol{x}_{i}\right|$, are introduced in order to keep the canonical structure of the evolution equations. It follows that:

$\partial_{t} J_{i}=\frac{\partial}{\partial \theta_{i}} \bar{H}, \quad \partial_{t} \theta_{i}=-\frac{\partial}{\partial J_{i}} \bar{H}$.

The dimensionless Hamiltonian is then given by:

$\bar{H}=-(4 N)^{-1} \sum_{m=1}^{N} \sum_{n=1, n \neq m}^{N} \ln G_{m n}$, 


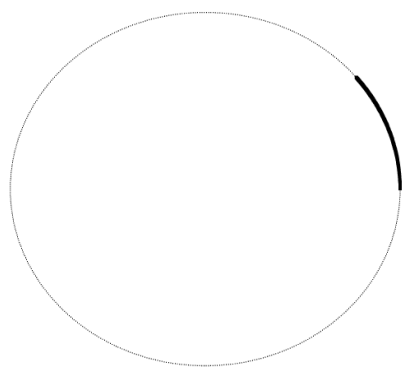

Fig. 2. Initial annulus of the distribution of vortices with $K=260$ and $e_{1}=0.108$.

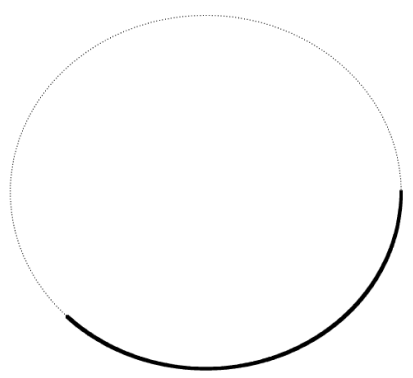

Fig. 3. Initial annulus of the distribution of vortices with $K=260$ and $e_{2}=0.624$.

where

$G_{m n}=\left[J_{m}+J_{n}-2 \sqrt{J_{m} J_{n}} \cos \left(\theta_{m}-\theta_{n}\right)\right]$.

Consider an initial distribution of vortices on an annulus: all vortices have the same radial coordinates $r_{s}=1$. The angular coordinates of every vortex are chosen as follows:

$$
\begin{aligned}
\theta_{s}= & 2 \pi N^{-1} e(s-1) \Theta(K-s)+ \\
& 2 \pi N^{-1}(1-e)(s-1) \Theta(s-K),
\end{aligned}
$$

in which $s=1,2, \ldots, N$ and $1<K<N$. The first vortex has the angular coordinate $\theta_{1}=0$. The set of the first $K$ vortices is distributed with a spacing of $2 \pi N^{-1} e$, all the others are distributed with a spacing of $N^{-1}(1-e)$. Here, $0<e<1$. The function $\Theta(z)$ is Heaviside's function, $\Theta(z)=1$ when $z>0$ and $\Theta(z)=0$ when $z<0$. In this way, the initial distribution of vortices is not symmetric. However the kinetic moment, one of the motion integrals, $\bar{P}$, defined by $\bar{P}=-\frac{1}{2} \sum r_{i}^{\prime 2}$, as well as the enstrophy $Z_{2}$ and other moments are unchanged for different values of the parameters $K$ and $e$.

The Hamiltonian of the system is a function of the parameters $e, K: H=H(e, K)$. This Hamiltonian being conserved, it is given by the initial value, $H_{0}$ which is calculated from an initial vortex distribution :

$H_{0}(e, K)=-(4 N)^{-1} \sum_{i=1}^{N} \sum_{j=1, j \neq i}^{N} \ln \left(1-\cos \theta_{i j}\right)$.

$\theta_{i j}=\theta_{i}-\theta_{j}$ is calculated from Eq. (2). In this expression, only terms depending on angles appear.

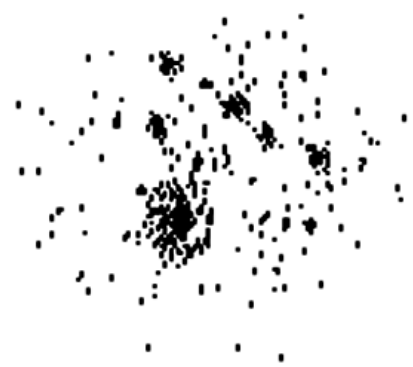

Fig. 4. Distribution of vortices at $t \sim 50$ with $K=260$ and $e_{1}=0.108$.

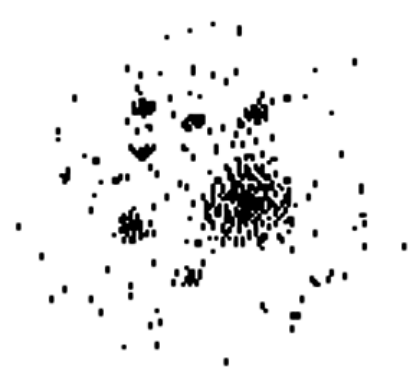

Fig. 5. Distribution of vortices at $t \sim 50$ with $K=260$ and $e_{2}=0.624$.

Moreover, the circulation $\Gamma$ is fixed by the number of vortices $N$. In the structure of the model, all other motion integrals $Z_{i}$ (enstrophy $Z_{2}, \ldots$ ) are automatically conserved. For instance let $N=750$ and $K=260$. The Hamiltonian $H(e, K)$, with fixed $K$ is a function of $e$ only. The dependence $H=H_{K}(e)$ allows to graphically find $e_{1}$ and $e_{2}$ for a given value of $H$ (see Fig. 1). For example let the initial value $H_{0}=0,25$. Then, for $K=260$, the corresponding values are $e_{1}=0,108$ and $e_{2}=0,624$ (see Figs. 2 and 3).

The evolution of a vortex distribution for a system with the given initial conditions has been numerically studied by using a 4th order Runge-Kutta scheme. The conservation of the energy $\mathrm{H}$ and of the angular momentum $\mathrm{P}$ has been checked during the calculations. The analysis shows that parameters have small variations with errors defined by $\left|H_{i}-H_{0}\right| /\left|H_{0}\right| \leq 3 \cdot 10^{-3}, \quad\left|P_{i}-P_{0}\right| /\left|P_{0}\right| \leq 2 \cdot 10^{-7}$. The iteration process has been performed from $t=0$ to $t=50$ which corresponds to 50 reference cycles. Although quadratic difference being relatively significant for the Hamiltonian which could lead in principle to intensification of a mixing process because of the accumulation of numerical errors, no visible tendency to a gradual homogenization has been observed.

The final results of calculations (for $t \geq 50$ ) are presented in Figs. 4 and 5. The time at $t \sim 50$ agrees with the results presented in the experiment of Huang and Driscoll (1994); Fine et al. (1995) (Fig. 6). According to the experiments, a formation of radically different configurations has already been observed for $t \sim 60$ and this justify our compilations.

Figures 4 and 5 show that vortex distributions having different initial distributions and characterized by identical global invariants, do not evolve to an universal axially 


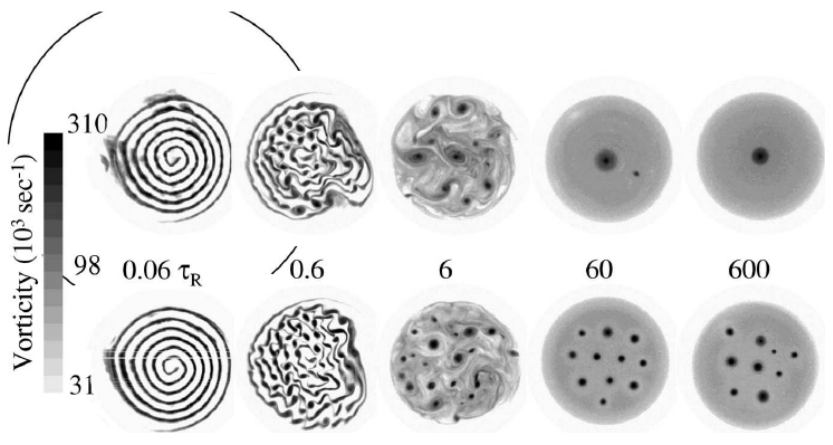

Fig. 6. "Vortex crystals" observed in the experiments of Huang and Driscoll (1994); Fine et al. (1995).

symmetric distribution. It is observed that vortex "clusters" are auto-organized in different ways. This can be explained by the fact that the Hamiltonian contains a non-vanishing fluctuating part which affects the evolution processes in the weakly dissipating system. If this fluctuating part has to be disregarded, the system would evolve to the axially symmetric configuration. This observation is qualitatively confirm to the recent experiments on "vortex crystal" formation (Fine et al., 1995; Huang and Driscoll, 1994) (see Fig. 6). Indeed, in these experiments, it has been observed that a 2-D system evolving without dissipation and forcing (physical or numerical), does not forget the structure of the initial vortex distribution. These observations are correlated with our calculations and confirm qualitatively the hypothesis of Batchelor (1967) that a quasi-final state of a perfect vortex system is fixed by its history and "keeps in mind" its initial configuration.

Note also that a different final state can appear depending on the initial configuration: (a) "basic" states reaching a maximum vorticity in the center, (b) "vortex crystals", and (c) states where no strong vortices persist and which probably correspond to principle of "minimum enstrophy".

\section{Perturbations and formation of vortex clusters}

Let us now consider the evolution of a vortex gas in the presence of chaotic internal and external perturbations. Having in mind a possible comparison with experimental data, we choose a configuration where the vortices are placed in a spiral (cf Fig. 7) similar to the initial spiral distribution of vorticity in Fig. 6.

\subsection{Analytical consideration}

The dimensionless Hamiltonian (normalized with the characteristic spatial scale $R$ and the temporal one $\tau$ ) of a system composed of $N \gg 1$ point vortices is

$H=(2 N)^{-1} \sum_{i \neq j ; i, j=1}^{N} H_{i j}$.

Here, $H_{i j}$ is the Hamiltonian of the interaction between vortices $i$ and $j$. For point vortices in an infinite plane, the
Hamiltonian is $H_{i j}=-\ln r_{i j}$ and is characterized by a longdistance interaction. If $N \gg 1$, vortices are not only influenced by neighboring vortices, but in essence by all the vortices, including those far away. This observation allows one to conclude that: (a) each vortex of the system containing a great number of vortices, moves quasi-independently with respect to the motion of its neighbor; (b) the situation is as if the selected vortex was in a self-consistent field created by all the vortices, including those far away.

Let us introduce the expression $H_{i}=D^{-1} \int_{D} d \boldsymbol{x}_{j} H_{i j} \equiv\left\langle H_{i j}\right\rangle$. Here, $D$ is an integration domain with a dimension defined by the conservation of momentum. The expression $H_{i}$ can be interpreted as the Hamiltonian of the $i$ th vortex averaged with respect to positions of all other vortices. The average can be also carried out by using a probabilistic approach if one introduces $P\left(\boldsymbol{x}_{j}\right)$, which gives the probability of finding the vortex $j$ in the vicinity of $\boldsymbol{x}_{j}$. In this case, $H_{i}$ can be defined by $H_{i}=\int_{D} d \boldsymbol{x}_{j} P\left(\boldsymbol{x}_{j}\right) H_{i j}$. It is noticed that a concrete structure of $P\left(x_{j}\right)$ would require an additional special analysis; so, we limit ourselves to the simplest approximation when $P\left(\boldsymbol{x}_{j}\right)=D^{-1}$. Let us then introduce the operator $\widehat{M}_{i}[\ldots] \equiv\left(N^{-1} \sum_{j}-D^{-1} \int d \boldsymbol{x}_{j}\right)[\ldots]$. After some mathematical manipulations, the Hamiltonian (3) takes the following form:

$$
\begin{aligned}
H & =\sum_{s=1}^{N} H_{s}+\frac{N}{2} \widehat{M}_{i} \widehat{M}_{j}\left(H_{i j}-\left\langle H_{i j}\right\rangle\right) \\
& \equiv \sum_{s=1}^{N}\left(H_{s}+\underline{\left.\frac{1}{2} \widehat{M}_{i} \widehat{M}_{j}\left(H_{i j}-\left\langle H_{i j}\right\rangle\right)\right)} .\right.
\end{aligned}
$$

The first term of the Hamiltonian defined by Eq. (4) describes the collective effects of the energy, which dominate when $N \gg 1$. According to the definition, $H_{s}$ depends only on $r_{s}=\left|\boldsymbol{x}_{s}\right|$. The second term describes stochastic fluctuations caused by individual interactions between vortices. These effects are obviously significant near a peripheral zone of the vortices' distribution. If $N \gg 1$, the last term of Eq. (4) is small. Indeed when $N \gg 1$, (a) the averaged quadratic deviation of $H\left(r_{i}\right)$ from the sum $N^{-1} \sum_{j=1}^{N} H_{i j}$ and (b) the operator norm $\widehat{M}_{i}$ are small. By replacing the exact expression $\frac{1}{2} \widehat{M}_{i} \widehat{M}_{j}\left(H_{i j}-\left\langle H_{i j}\right\rangle\right)$ by its value averaged with respect to the motions of all other (except $i$ ) vortices, $W\left(\boldsymbol{x}_{s}\right)$, we partly simplify the Hamiltonian. Such a procedure is similar to the one of Midgal (1975), p. 158, when one passes from the "multi-particles" description to the "mono-particle" description. According to its definition, the function $W\left(\boldsymbol{x}_{s}, t\right)$ has a zero average value $\left\langle W_{s}\right\rangle$, i.e. it is a oscillating function with respect to angle $\theta_{s}$ and/or with respect to time (see Sect. 2). The function $W_{s}$ preserves all the information about an initial distribution of vortices.

So, the problem reduces to a problem of a motion of a "particle" with a unit "mass" in a self-consistent field $H_{s}\left(r_{s}\right)+W_{s}\left(r_{s}, \theta_{s}, t\right)$. For simplicity of the notation, we will further omit the index $i$, considering the motion of a test vortex. We use the variables defined by the transformation 
$(x, y) \rightarrow(\theta, J)$, with $x=\sqrt{2 J} \sin \theta$ and $y=\sqrt{2 J} \cos \theta$ (such a transformation is largely used in some nonlinear problems). The equations of motion of the test vortex become

$$
\begin{aligned}
& \partial_{t} J=-\partial_{\theta} H=-\partial_{\theta} W, \\
& \partial_{t} \theta=\partial_{J} H=\Omega(J)+\partial_{J} W .
\end{aligned}
$$

If there is a stochastic disturbance in the Hamiltonian, internal and external $W_{s}\left(r_{s}, \theta_{s}, t\right) \neq 0$, it can be assumed that a vortex trajectory consists of two parts: regular and fluctuating (we follow here the idea formulated for Kapitsa's pendulum, see Kapitsa, 1951). For regular variables averaged with respect to stochastic fast fluctuations, the procedure of averaging leads to:

$$
\begin{aligned}
\partial_{t} \bar{J} & =-\overline{\partial_{\theta} H} \\
& =-\overline{\theta_{1} \partial_{\theta \theta} W(\bar{J}, \bar{\theta})}-\overline{J_{1} \partial_{\theta J} W(\bar{J}, \bar{\theta})}+\ldots, \\
\partial_{t} \bar{\theta} & =\overline{\partial_{J} H} \\
& =\Omega(\bar{J})+\overline{\left.J_{1} \partial_{J J} W(\bar{J}), \bar{\theta}\right)}+\overline{\theta_{1} \partial_{J} W(\bar{J}, \bar{\theta})}+\ldots
\end{aligned}
$$

Subtracting one equation from the other, we obtain for $J_{1}=J-\bar{J}$ and $\theta_{1}=\theta-\bar{\theta}$

$$
\begin{aligned}
& \partial_{t} J_{1}=-\partial_{\theta} W(\bar{J}, \bar{\theta})+\ldots \\
& \partial_{t} \theta_{1}=J_{1} \partial_{J} \Omega(\bar{J})+\partial_{J} W(\bar{J}, \bar{\theta})+\ldots
\end{aligned}
$$

We look for solutions in the form $W \sim U e^{-i \omega t}, J_{1}, \theta_{1} \sim e^{-i \omega t}$ :

$$
\begin{aligned}
J_{1} & =\frac{1}{i \omega} \partial_{\theta} U(\bar{J}, \bar{\theta}), \\
\theta_{1} & =7-\frac{1}{i \omega} \frac{1}{i \omega} \partial_{\theta} U(\bar{J}, \bar{\theta}) \partial_{J} \Omega(\bar{J})-\frac{1}{i \omega} \partial_{J} U(\bar{J}, \bar{\theta}) .
\end{aligned}
$$

Substituting these expressions in Eq. (6), omitting the imaginary terms (field variables are real; appearance of the imaginary number $i$ is equivalent to a difference in phase of a harmonic function of $\pi / 2$ ) and putting in the $1 / 2$ factor (average of a harmonic function on the period), we find

$$
\begin{aligned}
\partial_{t} \bar{J} & =-\overline{\partial_{\theta} H}=-\frac{\Omega^{\prime}}{2 \omega^{2}} \partial_{\theta} U \partial_{\theta \theta} U+\ldots \\
& =-\partial_{\theta}\left(H(\bar{J})+\frac{\Omega^{\prime}}{4 \omega^{2}}\left(\partial_{\theta} U\right)^{2}\right), \\
\partial_{t} \bar{\theta} & =\overline{\partial_{J} H}=\Omega(\bar{J})+\frac{\Omega^{\prime}}{2 \omega^{2}} \partial_{\theta} U \partial_{J \theta} U+\ldots \\
& =\partial_{J}\left(H(\bar{J})+\frac{\Omega^{\prime}}{4 \omega^{2}}\left(\partial_{\theta} U\right)^{2}\right)+\ldots
\end{aligned}
$$

Here, $\Omega^{\prime} \equiv \partial_{J} \Omega(\bar{J})$.

We can define the "effective" Hamiltonian as

$$
\begin{aligned}
H_{\mathrm{eff}} & =H(\bar{J})+\left(\Omega^{\prime} / 4 \omega^{2}\right)\left(\partial_{\theta} U\right)^{2} \equiv \\
& =H+H^{\prime}=H\left(r_{i}\right)+\varepsilon V\left(r_{i}, \theta_{i}, t\right) .
\end{aligned}
$$

Note that this is equivalent to the situation where a test vortex moved along an averaged trajectory defined by the averaged Hamiltonian $H_{\text {eff }}$ which is a coordinate dependent function. The disturbance of a fundamental state is characterized by a small non-dimensional parameter $\varepsilon \ll 1$. By definition, $V(r, \theta, t)$ is a periodic function with respect to $\theta$, $V(r, \theta, t)=V(r, \theta+2 \pi, t)$ and can be expanded into a Fourier series:

$V(r, \theta, t)=\frac{1}{2} \sum_{m}\left[V_{m}(r, t) e^{i m \theta}+c . c.\right]$.

Here, the abbreviation $c . c$. represents the complex conjugation. The disturbance $V$ is a real function. For this reason, both positive and negative $m$ are present in the sum (11): the Fourier coefficients satisfy the condition $V_{m}=V_{-m}^{*}$.

Then Eq. (9) reduced to

$$
\begin{aligned}
\partial_{t} J & =-\frac{1}{2} \varepsilon \sum_{m}\left(i m V_{m}(t) e^{i m \theta}+c . c .\right), \\
\partial_{t} \theta & =\Omega(J)+\frac{1}{2} \varepsilon \sum_{m}\left(\left(\partial_{J} V_{m}(t)\right) e^{i m \theta}+c . c .\right) .
\end{aligned}
$$

When the fluctuation is neglected $(\varepsilon=0)$, the solutions are $J(t)=J^{0}, \theta(t)=\Omega\left(J^{0}\right) t+\theta^{0}$, with $\theta^{0}$ being the initial phase.

Let us consider the case where disturbances are localized near a level $J_{0}$, near when $H^{\prime}$ has a distinct maximum, i.e. the excitation is important near this level. In this case the derivative $\partial_{J} V_{m} \mid J_{0}=0$. We can simplify Eq. (12) near $J_{0}$ by putting $r_{1}=r-r_{0}$, with $\left|r_{1}\right| \ll r_{0} \equiv \sqrt{2 J_{0}}$, and keeping only principal terms in the right-hand side of the equations. Taking $\theta=\theta_{1}+\Omega\left(J_{0}\right) t$, we obtain

$$
\begin{aligned}
\partial_{t} r_{1} \simeq & -\varepsilon\left(2 r_{0}\right)^{-1} \times \\
& \times \sum_{m}\left[i m V_{m}\left(r_{0}, t\right) e^{i\left(m \Omega\left(r_{0}\right) t+m \theta_{1}\right)}+c . c .\right], \\
\partial_{t} \theta_{1}= & \Omega(r)-\Omega\left(r_{0}\right)+\ldots=r_{0} \Omega^{\prime}\left(J_{0}\right) r_{1}+\ldots .
\end{aligned}
$$

We suppose now that the conditions of the problem are such that there is only one, a governing term, in the sum on $m$. The simplified Eqs. (13) for this spectral component become

$$
\begin{aligned}
& \partial_{t} r_{1}=-i \varepsilon\left(2 r_{0}\right)^{-1} m V_{m}(t) e^{i\left(m \theta_{1}+m \Omega\left(r_{0}\right) t\right)}+c . c . \\
& \partial_{t} \theta_{1}=r_{0} \Omega^{\prime}\left(J_{0}\right) r_{1} .
\end{aligned}
$$

Since $V_{m}(t)$ is dependent on time it can be expanded into a Fourier series of the form $V_{m}(t) \sim \sum_{n} V_{m n} \exp [-i n \gamma t]$. It can be shown that the term in the resulting sum with respect to $n, V_{m n} \exp \left[-i\left(m \Omega\left(J_{0}\right)-n \gamma\right) t\right]$ that satisfies the condition $\Delta_{m n}=m \Omega\left(J_{0}\right)-n \gamma \rightarrow 0$, is the most important (see also Chirikov, 1969, 1978, 1979; Zaslavskii and Sagdeev, 1988), and hence is the only term that need be kept. In this case $\left|r_{1}\right| \sim \varepsilon / \Delta_{m n}$ and when $\Delta_{m n}=m \Omega\left(J_{0}\right)-n \gamma \rightarrow 0$, and $\varepsilon \neq 0$, the magnitude of $r_{1}$ can be significant.

The complex coefficient $V_{m n}$ is written in the form $V_{m n}=|V| \exp (i \varphi)$. Introducing a new variable, $\psi=m \theta_{1}+\Delta_{m n} t+\varphi+\pi$, one obtains

$\partial_{t} r_{1} \simeq-\varepsilon m r_{0}^{-1}|V| \sin \psi$

$\partial_{t} \psi=\Delta_{m n}+\left.m r_{0} \Omega^{\prime}\right|_{r_{0}} r_{1}$,

by neglecting the terms of high order. The system (15) can be put in the canonical form

$\partial_{t} r_{1}=-\partial_{\psi} \tilde{H}, \partial_{t} \psi=\partial_{r_{1}} \tilde{H}$, 


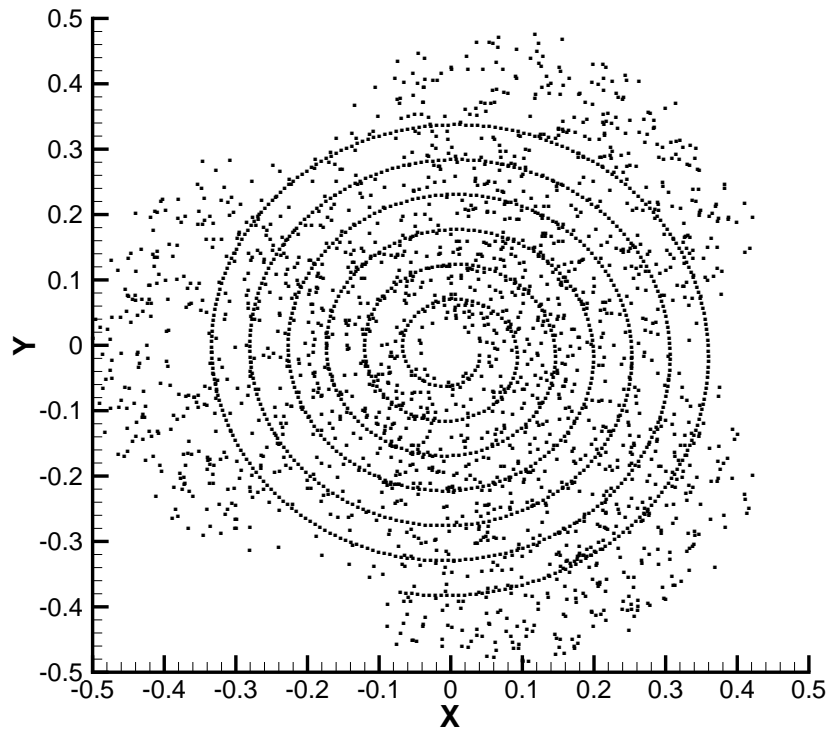

Fig. 7. Observation of 3 "super vortices", zones which trap localized vortices. The initial vortex distribution on the spiral is shown.

with the Hamiltonian

$\tilde{H}=\left.(1 / 2) m r_{0} \Omega^{\prime}\right|_{r_{0}}\left(r_{1}\right)^{2}-\varepsilon m|V|\left(r_{0}\right)^{-1} \cos \psi+\Delta_{m n} r_{1}$.

Moreover (15) are isomorphous to the non linear pendulum equations $\ddot{\psi}+\Omega_{N}^{2} \sin \psi=0$, with $\Omega_{N}^{2}=m^{2} \varepsilon\left(r_{0}\right)^{-1}|V|$ $\left.r_{0} \Omega^{\prime}\right|_{r_{0}}$. The different aspects of the theory and applications of a non linear oscillator model to laden particles in a plasma are given in the above cited works. Equilibrium positions correspond to points $\psi_{k}=k \pi$, where $k=0, \pm 1, \pm 2, \ldots$ If $k$ is even or zero, i.e. $\psi_{k}=0, \pm 2 \pi, \pm 4 \pi, \ldots$, the corresponding points are points of a stable equilibrium of the pendulum. If $k$ is uneven, i.e. $\psi_{k}= \pm \pi, \pm 3 \pi, \ldots$, the corresponding points are hyperbolic, i.e. points of unstable equilibrium. Through these points pass the "separating lines". The transformation of $\theta \rightarrow \psi$ means that we have passed the rotation reference, $\psi=m_{0} \theta+\varphi+\pi=m \theta_{1}+\Delta_{m n} t+\varphi+\pi$. If $\Delta_{m n}=0$, the points of the equilibrium positions in this rotation reference are defined by the expression $m_{0} \theta_{1 k}=k \pi+C^{t e}$ (c is an arbitrary constant). The stable equilibrium positions are given by $\theta_{1 k}=k \pi / m_{0}$, with $k$ even and the unstable equilibrium positions are given by $\theta_{1 k}=k \pi / m_{0}$, with $k$ uneven. The possible trajectories of a test vortex are topologically different near the stable and unstable equilibrium points. In the first case, these lie on closed orbits, where the situation is as if vortices are kept near these points.

The result of qualitative analysis shows that the theory is valid if

$0<\varepsilon \ll \delta \ll 1 \ll 1 / \varepsilon$,

where $\delta=\Omega_{0}^{-1} r_{0} \Omega_{0}^{\prime}$, with $\Omega_{0}=\left.\Omega\left(r_{0}\right) \equiv\left(r^{-1} \partial_{r} H\right)\right|_{r_{0}}$, which can be approximated by $\Omega_{0} \sim r_{0}^{-2} H_{0}$ because there is no specific scale.

This simple analysis permits one to establish conditions and possible scenarios for the self-organization of point vortices into clusters which become apparent in observations

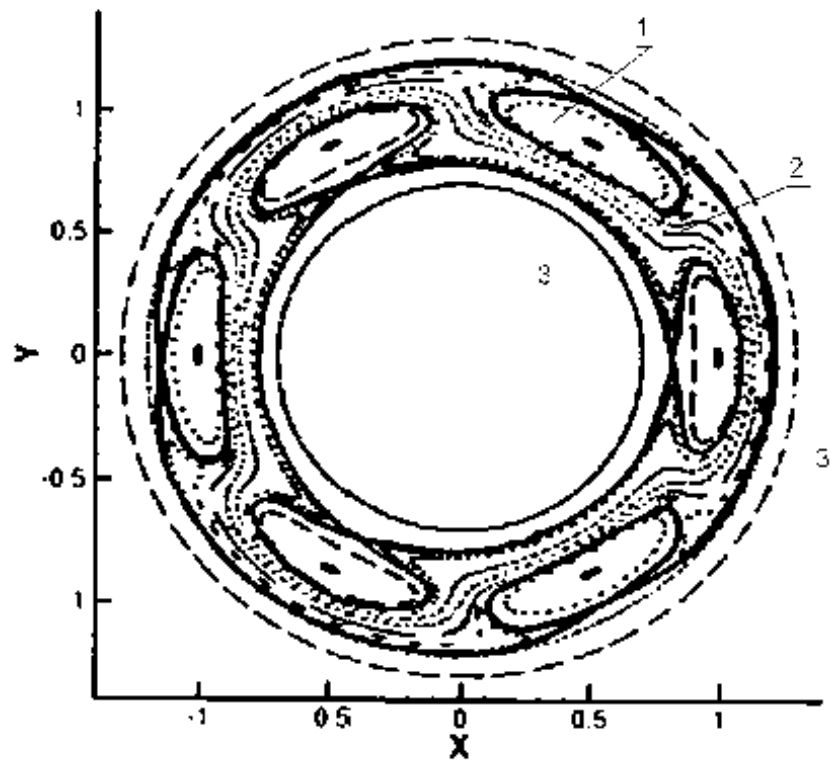

Fig. 8. The numerical calculation showing the self-organisation of 2 -D vortices into "super-vortices". The vortices, which are in the disturbed domain, stay but others circumvent these zones.

of "super-vortices". An example of such a situation is the effect of a regular structure formation, similar to observed "vortex crystals", which would occur when $m_{0} \neq 0$ (there is a angular inhomogeneity in a initial distribution of vortices), $\partial_{J J} H_{S}(J) \neq 0$ and the stochastic fluctuating part of the Hamiltonian, which is essentially localized on the circle, varies quickly in the test time, $\varepsilon \sim \Omega^{\prime}(J) / \omega^{2} \ll 1$.

\subsection{Numerical confirmation}

These remarks agree with the numerical calculations (Figs. 7 and 8) based on the discussed equations. For calculations, we took $N=750$ test vortices initially accommodated on a 6 spiral branch with a maximal radius $R=0,375$. We used a "rough function" $\left.V=\left(1-\cos 3 \theta_{i}\right)\right) \exp \left[-\left(r_{i}-r_{0}\right) / b^{2}\right]$ with $b=0,1, \varepsilon=0.5$. The corresponding result is shown in Fig. 7. The second test has been performed with the function $V=\cos 3 \theta_{i} \exp \left[-\left(r_{i}-r_{0}\right) / b^{2}\right]$, for 16 test vortices and the parameters $r_{0}=1, b=0,1, \varepsilon=0,5$ (Fig. 8). The numerical results show that a vortex initially localized in a region of "capturing", will be enclosed in this area (1). The region of "capturing" is defined from a minimum of the potential, $V$. These regions are separated from the rest of the area by a "separating line". There are a few possibilities: (a) point vortices localized initially in the domain 1, cluster together forming "super-vortices"; (b) vortices initially in domains (2) and (3) move stochastically, penetrating from (2) into (3) via the hyperbolic points (zones). The domains (2) and (3) finally form a quasi-homogeneous patch having a radius $r_{f}>r_{\text {init }}$ of a relatively small concentration of vortices, while domains with a higher concentration of vortices will be organized in domains (1). 


\section{Discussion}

The present work was motivated by a few reasons and observations.

Experiments by Huang and Driscoll (1994); Fine et al. (1995) on 2-D freely evolving turbulence without forcing and dissipation show that the relaxation of a turbulent system to a basic state can be sometimes arrested (Fig. 6). The formation of regular vortex lattices ("vortex crystals") can be observed. These "vortex crystals" are composed of 5-20 individual "super-vortices" with an intensity 4-6 times larger than the surrounding vorticity. This regular structure rotates in conjunction with the background and does not undergo any deformation while the time $\geq \sim 10^{4}$ times larger than characteristic reference time.

The experiments have been conducted in electronic plasma placed in a very strong magnetic field. In such a situation, the system can be described in the framework of the model of point vortices.

Really, the motion of individual electrons include fast and slow components. First, the "heated" electrons move along of the magnetic field lines (axis $z$ ). In presence of an energetic "closure fitting" on the dispositive ends, "heated" electrons are submitted to successive reflections from these closure fittings with a characteristic time $\tau_{t h} \sim L / v_{t h} \sim T^{-1 / 2}$. The electrons follow in the same time circular trajectories around field lines, $\boldsymbol{B}$, with a characteristic time (of Larmor) $\tau_{L} \sim \omega_{L}^{-1} \sim B^{-1}$. Finaly, they participate in a collective motion which is a rotating macroscopic collective motion around the axis $z$ whose characteristic time is $\tau_{\mathrm{col}} \sim B / E$. If the fields are intense, one can assume the condition $\tau_{\mathrm{col}} \gg \tau_{t h}, \tau_{L}$. Let $\Delta t$ be an exposure time of observation on the monitor (Fig. 9) that satisfies the condition $\tau_{\mathrm{col}} \gg \Delta t \gg \tau_{t h}, \tau_{L}$. In this case, the averaging with respect to fast motions permits one to eliminate all $z$-dependencies of fields from the consideration.

After averaging with respect to fast motions along the magnetic field and, in this way, "smearing" the electric charge in a spatial domain of volume $V \sim a^{2} L$, this electron system is similar to a system of $N$ macroscopic charged "bars", $L \sim 1 \mathrm{~m}$, of very small diameters $a$ (Larmor's radius, $a \sim 5 \mu \mathrm{m}$ ) aligned with the magnetic induction, $\boldsymbol{B}$, and moving on a timescale of the order of the macroscopic time without changing their orientation. The "bars" never touch; in fact there are no contacts between electrons. The characteristic time, $\tau_{R}$ (the turnover time), of the collective azimuthal motion of the "bars" (of electrons whose fast longitudinal motions have been averaged), is macroscopically great.

If $a / L \ll 1$, i.e. $a \rightarrow 0$, the effects containing the factor $a / L$ can be neglected.

The coordinates of these "bars" in the plane perpendicular to the magnetic field are $x_{i}, y_{i}, i=1, \ldots, N$. We obtain thus the two-dimensional system whose motion in the $x-y$ plane is controlled by equations isomorphous to the equations of motion of two-dimensional point vortices (with the Hamiltonian - energy of interaction expressed in terms of canonical

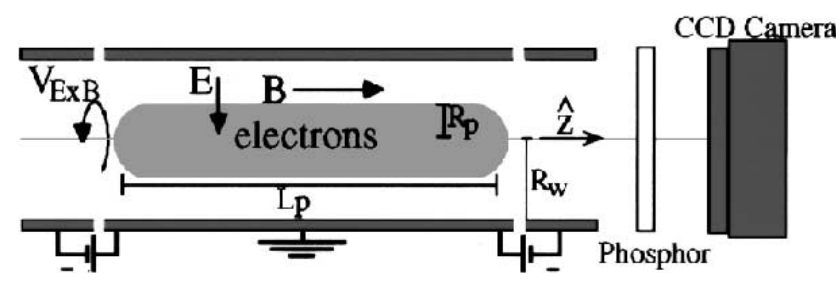

Fig. 9. Experiment of plasma: scheme of experimental apparatus.

variables - depending logographically of distances between the "bars").

The integral quantities which can be assimilated with energy, enstrophy, etc, measured in these experiments for the sequences leading to the different final configurations (Fig. 6), do not change during the experiments, i.e. the system is not dissipative.

These qualitative arguments explain why we choose the model of point vortices. In the framework of our model and in the presence of stochastic fluctuations and in absence of a dissipation, the clustering of point vortices can stop due to the spontaneous formation of regular dynamical vortex structures.

The observed regular structure can be explained without incorporating special physical arguments beyond the description. A good qualitative agreement between results of our analysis and the experiments concerning the evolution of the great number of "vortices" was observed.

The numerical simulations showed that different initial configurations of vortices having identical dynamical invariants, do not evolve to an universal axially symmetric distribution. This can be explained by the fact that the fluctuating part of the Hamiltonian, which can be neglected in some experiments and numerical studies, plays an important role in the study.

As concluding remarks, let us present some explanations and give useful references (see also Miller et al., 1992; Danilov and Gurari, 2000; Pavlov et al., 2002) concerning some numerical approaches to the problem of the 2-Dturbulence evolution.

Two-dimensional flows of an incompressible fluid is usually described by the evolution equation for vorticity, having the form $\partial_{t} \Omega+[\Omega, \psi]=F+D$. Here, all notations are standard, the vorticity field can be both continuous as for the most part of works and discontinuous as in our model, $F$ the forcing, and $D$ the dissipation. If $F=0$, or if, after averaging, a fluctuating part of the forcing can be grouped with the Hamiltonian (Sect. 3), $H \rightarrow H_{\text {eff }}$, the omission of dissipative terms in the governing equations signifies that we consider the effects of formation of dynamic regular structures at an initial stage of decaying turbulence (see further) when viscous effects have not yet become apparent.

In numerical analysis when $F \neq 0$, and $D \neq 0$, the forcing term is usually localized in the vicinity of a small space scale (large wave number $k_{f}$ ), and the dissipation operator $D$ combines frictional and viscous terms. The latter usually contains 


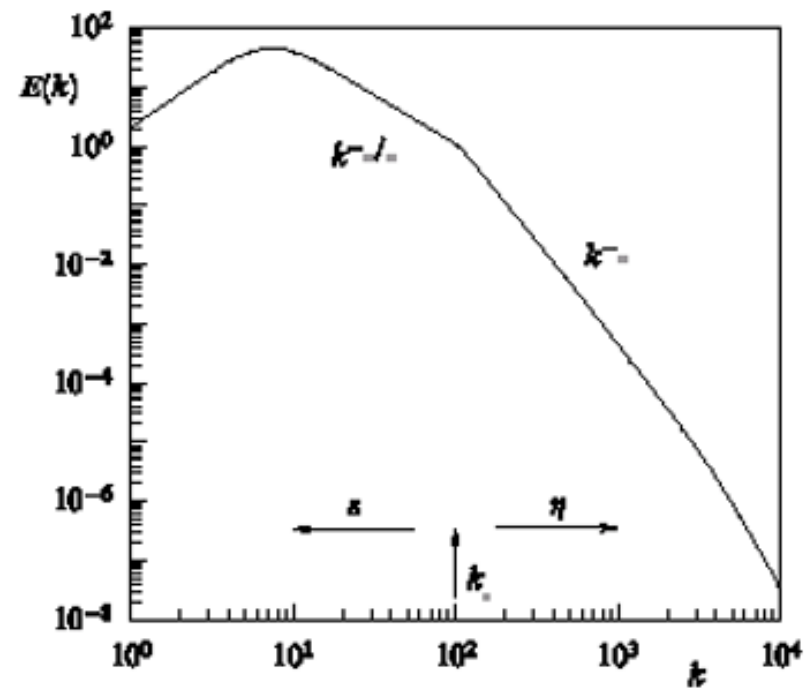

Fig. 10. Schematic view of the kinetic energy spectrum of 2-D turbulence. $k_{f}$ is the forcing wave-number. On the right is the enstrophy interval characterized by the enstrophy flux $\eta$, which transforms into the enstrophy dissipation range for large $k_{d}=\left(\eta / v^{3}\right)^{1 / 6}$ (Newtonian fluid). On the left is the energy interval characterized by the energy flux $\epsilon$.

the classical viscous term, $D=v \Delta \Omega$, with a constant coefficient of viscosity $v$ (Newtonian fluid), but may include other dissipative effects. For computational purposes, one often applies the so-called hypofriction and hyper-viscosity rule, $D=\left((-1)^{n+1} \lambda_{n}(-\Delta)^{-n}+(-1)^{m+1} v_{m} \Delta^{m}\right) \Omega$. The $\lambda_{n^{-}}$ term serves to suppress the upscale energy cascade, the $v_{m}$ viscosity halts the downscale enstrophy cascade. For instance, the natural physical dissipation in 2-D or quasi-2D flows is due to bottom friction in geophysical applications, which correspond to $n=0$ (Ekman friction). Such friction equally damps all modes, with large and small space scales. Hypofriction with a negative power for the Laplacian in $D(n>0)$, was proposed for a turbulent phenomenology. It selectively suppresses the greatest modes of the system and recreates a dissipation-free (inertial) interval in the energy range, but it has no direct physical relevance. It has the advantage that the inertial (enstrophy) interval may be pushed to higher $k$ without increasing the computational grid.

The evolutional equation can be written in the equivalent form $\partial_{t} \Omega+\{\Omega, \delta H / \delta \Omega\}=F+D$ where the functional Poisson bracket, $\{.,$.$\} is introduced (see Goncharov and Pavlov,$ 2001; Pavlov et al., 2002, 2001).

In the absence of forcing-dissipation, the equation conserves a few integrals: kinetic energy, $E$, enstrophy, $Z_{n}$. Furthermore, this evolutional equation gives rise to an infinite set of conserved integrals, called Casimirs (moments $Z_{n}$, isolevel areas of vorticity in the $x y$-plane, etc).

The basic equation for a flat domain is usually solved by pseudo-spectral methods. In the Fourier representation (discrete or continuous), this equation takes on the form $\partial_{t} \Omega_{\mathbf{k}}+J_{\mathbf{k}}=D_{\mathbf{k}}+F_{\mathbf{k}}$. Here, $J_{\mathbf{k}}$ denotes the $\mathbf{k}$ th Fourier mode of the Jacobian $J(\Omega, \psi)$, i.g. of $\{\Omega, \delta H / \delta \Omega\}$ which in spec- tral methods is implemented via fast Fourier transform (going back and forth between the $\boldsymbol{k}$ and $\boldsymbol{x}$-spaces, and replacing convolutions with products). One looks for the spectral energy density $E(k, t)$.

Forced 2-D turbulence can attain a statistically stationary state (Fig. 10), if the energy and enstrophy injected by a source are balanced by the dissipation.

The decaying case, when $F=0$ and $D \neq 0$, seems natural and requires no large-scale artificial dissipation. In some limiting regime (Batchelor, 1969) postulated the energy spectrum of the decaying turbulence to evolve according to the law $E(k, t)=E^{3 / 2} t f\left(E^{1 / 2} k t\right)$ defined in terms of a single parameter - the total (nearly conserved) energy $E$ - and the dimensionless function $f$ (of the only possible dimensionless combination of $E, k$ and $t$ ). By the same argument we could get the decay law for the total enstrophy $Z_{2} \sim t^{-2}$. Once again, the dimensional arguments would give a slope of -3 for the energy spectrum of the decaying turbulence at large $k$.

Unlike the 3-D case, one has only very limited experimental verification of the 2-D turbulence laws. One could simulate it (to some extent) in the laboratory environment, but only within a limited range of scales. Therefore, the bulk of 2-D-turbulence results were obtained in numerical simulations, with somewhat tenuous and speculative links to experiments and observation.

There are also analytic theories, advanced in the $60 \mathrm{~s}$ and 70s and based on certain closure assumptions. Their premises, however, are also hard to verify experimentally or numerically.

Let us add some useful references for both numerical experiences and modal spectral calculations on the decaying turbulence (see Danilov and Gurari, 2000).

McWilliams (1984) has shown decaying turbulence to evolve into long-lived coherent vortices, which persist for many turnover periods. The first examples of coherent vortices in decaying turbulence appeared in the early papers of Fornberg (1977); Basdevant et al. (1981), but McWilliams demonstrated this phenomena in different systems and for various initial conditions. Figure 11 shows a typical vorticity field of decaying turbulence.

The paper of McWilliams (1984) takes an initial spectrum $E(k, 0)$ with a slope of -3 at large $k$, and resolves the system on a $256^{2}$ grid. As the system evolves its spectrum steepens to -5 , and the enstrophy transfer drops to zero. But the vorticity kurtosis shoots from the initial Gaussian value of 3 to several dozen. Vortices form at intermediate scales (between the initial state and the box size).

The vortices can slow down the cascade processes (see McWilliams, 1990b), since they carry the bulk of enstrophy, but do not stretch and filament one another. Paper of Santangelo et al. (1989) made a systematic study of decaying turbulence and its spectra in an attempt to reconcile the multitude of reported spectral slopes. It uses a high resolution 1024-grid and long time integration of the initial Gaussian field of zero mean value and the initial energy spectrum $E(k, 0) \sim k\left[1+\left(k / k_{0}\right)^{\gamma+1}\right]^{-1}$ for $k_{0}=6$, and $\gamma=6$. 
The initial evolution creates vortex filaments via stretching by the large-scale velocity field. They carry over small-scale eddies as passive tracers, hence developing a slope -3 in the enstrophy range. At the next stage large coherent vortices evolve from the local vorticity extrema, and start breaking down into smaller size vortices. Due to two different mechanisms of vortex formation, no universal distribution of vortices by size and intensity appears as evident in their spectra.

The energy spectra have an interval of steep slope at small $k$, and a shallower (closer to -3 ) interval in the small vortices range. The total energy remains nearly constant during the evolution, while the enstrophy declining does not, however, drop to zero. Indeed, large-scale stable vortices lock up a sizable fraction of enstrophy, and will not let it cascade to small scales.

The main conclusion of Santangelo et al. (1989) is that the -3 spectrum could appear only at an intermediate stage of the process. Large vortices destroy scale invariance and steepen the low-mode spectra. Besides, the paper claims that the resulting spectral shape strongly depend on the initial state of the system. In particular, an initially steep spectrum produces $\pi / k_{0}$-size vortices that dominate the future evolution of the system. Shallower initial spectra, like the -3 used by McWilliams (1984); Benzi et al. (1988), give a broad spectrum of vortex sizes. Paper of Santangelo et al. (1989) sets the borderline initial slope for the two patterns somewhere between -3 and -6 .

The appearance of coherent vortices in the decaying turbulence allows them to be studied as statistical vortex ensembles (Benzi et al., 1988; McWilliams, 1990a; Carnevale et al., 1991; Benzi et al., 1992; Weiss and McWilliams, 1993). For this purpose, one needs to select coherent vortices from the small-scale turbulent background. The simplest selection rule identifies regions of vorticity field that exceed a prescribed threshold (in terms of rms vorticity). Another census analyzes the determinant of the velocity gradient and seeks regions where it takes negative values. Paper of Benzi et al. (1988) claims that the two methods give similar results. The initial -3 spectrum (at 512-resolution) evolves to $k^{-4,3}$. The authors estimate the vortex contribution to the energy spectrum to have a slope of $-6+\alpha$, depending on the vortex size distribution. That yields an energy slope of -4.1 , close to the observed value. At the final stages of evolution the dynamics of coherent vortices can be well approximated by point vortices.

Paper by McWilliams (1990a) studies the characteristics of 2-D vortices, particularly their time evolution. It takes an initial state with the energy spectrum $E(k, 0)=k^{6}\left(k+2 k_{0}\right)^{-18}$, on a $450^{2}$ grid. The vortices are selected by comparison with the "ideal" vortex profile. Their number decays in time, as $N \sim t^{-0,71}$. The maximal vorticity decreases, but its mean absolute value over all vortices remains nearly constant. The mean vortex size grows as $t^{0,2}$, and the mean separation as $t^{0,4}$. The vortices maintain a nearly Gaussian profile, but unlike (Benzi et al., 1988) this profile is not universal. The difference between the two cases could be attributed to different initial conditions. Finally, the
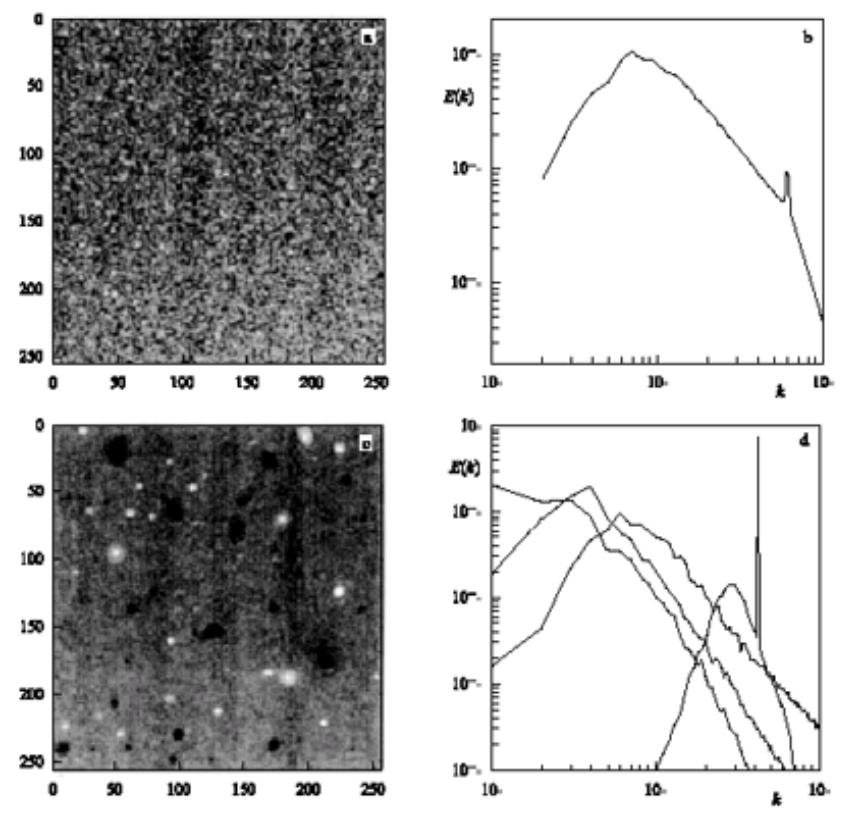

Fig. 11. Vorticity and energy spectra in stationary $(\mathbf{a}, \mathbf{b})$ and decaying (c, d) turbulence. (a) Realization of vorticity field at the quasistationary stage of evolution, (b) time-averaged energy spectrum, forced at wave numbers $k_{2} \in[58,62]$ and stabilized by the bottom friction; (c) Realization of vorticity field at the late stage of the decay process, $t_{v}=124$; (d) Evolution of the energy spectrum during the decay phase $\left(t_{v} \simeq 0,5,40,70,124\right)$. The initial spectral peak at $k \simeq 45$, and initial energy equals 1 .

enstrophy decreases as $t^{-0,4}$, in stark contrast to results of Batchelor (1969).

The prominent role of vortices in the decaying turbulence motivated the development of vortex models (Carnevale et al., 1991; Benzi et al., 1992; Weiss and McWilliams, 1993). Authors of these works assume that vortices behave like point vortices, at large separations, and each is determined by two parameters - the vortex radius and (uniform) vorticity level. When two vortices collide, that is come within a distance $1,7\left(R_{1}+R_{2}\right)$ of their radii, they merge into a single vortex of radius $\left(R_{1}^{4}+R_{2}^{4}\right)^{1 / 4}$. Such collisions conserve energy and decrease enstrophy, and thus can account for the enstrophy loss due to vortex straining and filamentation in real systems. Different initial conditions in papers by Carnevale et al. (1991); Benzi et al. (1992); Weiss and McWilliams (1993), however, lead to divergent results. Thus Benzi et al. (1992) has vortex sizes distributed initially according to the $R^{-3}$ law, which corresponds to the -3 spectrum of numerical simulations (Benzi et al., 1988). The terminal sizedistribution comes close to $R^{-2}$, which gives a -4 energy slope (close to -4.1 Benzi et al., 1988). The number of vortices decays as $t^{-0.6}$, which differs from the $t^{-0.7}$ law (McWilliams, 1990a), the $t^{-0.75}$ law (Carnevale et al., 1991), and the $t^{-0.72}$ law (Weiss and McWilliams, 1993).

The point-vortex dynamics analyzed by Carnevale et al. (1991); Weiss and McWilliams (1993), provides scaling laws for the vortex number, size distribution, distance distribution, 
and enstrophy, which agree with the pseudo-spectral results obtained by McWilliams (1990a). Based on the numeric results of McWilliams (1990a), particularly the conservation of average vorticity amplitude (over all vortices), Carnevale et al. (1991) proposed a hypothesis for decaying turbulence, consistent with the numerical observations.

Except energy conservation, as in the Batchelor theory (Batchelor, 1969), Carnevale et al. (1991) postulated the conservation of vorticity extrema. The latter follows naturally, when one views decay turbulence as the process of vortex merging. The enstrophy decay is confined to the vortex periphery (caused by filamentation), but it does not affect vortex cores. Assuming the conservation of vortex extrema $\Omega_{m}$ one could introduce the time and length parameters $\tau=\Omega_{m}^{-1}, l=\sqrt{E} / \Omega_{m}$. Assuming further a power decay law for the number of vortices $N_{v}$ with an exponent $\xi: N_{v} \sim t^{-\xi}$, and writing the energy and enstrophy (confined in vortex cores) as $E \sim N_{v} R^{4} \Omega_{m}^{2}, \quad Z_{2} \sim N_{v} R^{2} \Omega_{m}^{2}$, one gets the mean vortex size to grow as $l(t / \tau)^{\xi / 4}$ (energy conservation), while the distance between vortices grows as $l(t / \tau)^{\xi / 2}$, and the enstrophy of the entire flow decays as $\Omega \sim \tau^{-2}(\tau / t)^{\xi / 2}$. Such conclusions also agree with the numerical studies.

The proposed scaling differs from the classical Batchelor results, as well as the selective decay theory (see for instance Salmon, 1988). The latter postulates that turbulent decay should minimize enstrophy, subjected to the energy constraint (Carnevale et al., 1992). This theory was applied to describe late stages of turbulent decay in papers of Mattaeus et al. (1991a,b) and others. Paper of Carnevale et al. (1992) shows selective decay to predict higher decay rates than numerical simulations, as it fails to account for the role of coherent vortex structures in slowing the decay process.

The validity and utility of pseudo-spectral methods for two-dimensional turbulence dominated by vortices has been questioned by Dritschel (1993). It has been argued that pseudo-spectral methods introduce significant numerical dissipation on the vortex periphery, thus giving a wrong description of vortex mergers and the resulting filamentation (see Legras and Dritschel, 1993). Paper of Mariotti et al. (1994) demonstrated that thin filaments on the periphery, subjected to strong hyper-viscous dissipation, bring about a sharp increase of the overall dissipation rate of vorticity. Furthermore, the hyper-viscosity could cause undue oscillations of iso-contours on the periphery of vortex cores. He proposed an alternative method of contour dynamics, augmented by the so-called surgery. It allows in principle a higher spatial resolution than pseudo-spectral methods and, hence, a broader spectral range. The dissipation scale (which cuts off fine structures) corresponds to resolution 7000 in pseudospectral methods. The paper finds that the vortex size distribution is not self-similar, and steepens as the system evolves. The corresponding energy spectra vary from nearly $k^{-5}$ at large scales to $k^{-3}$ at small ones. It also finds that some other characteristics, like the growth rate of vortex sizes for large vortices and the decay rate of enstrophy, are markedly different from the pseudospectral results. The reason for such a departure, however, is not only the overall decrease of dissi- pation, as claimed by the author, but may include other factors, such as sharp boundaries of the vortex patches, in the contour dynamics.

Let us stress that the entire decay process is due to the enstrophy dissipation at short wavelengths. Without such dissipation, the system would relax to a statistical equilibrium state with an equipartition energy spectrum $k^{-1}$ (see, for instance, a paper of Holloway, 1986). One could expect the numerical dissipation to be equally important. Indeed, the key process of large scale condensation (vortex merger) is largely determined by small-scale dissipation.

The criticism about in respect of pseudo-spectral methods (see paper of Dritschel, 1993) is based on the notion of a well identified (sharp) vortex boundary, while these methods operate with smooth fields, without jumps. It is not clear to what extent vortex patches could represent smooth fields. On the other hand the different behavior of pseudo-spectral decaying turbulence from that of contour dynamics could be interpreted as a difference in initial conditions, in the spirit of the paper of Santangelo et al. (1989).

Papers of Chasnov (1997); Bartello and Warn (1996); Chasnov and Herring (1998) describe the dependence of the decay characteristics on the enstrophy dissipation mechanism. The main result is that one should not expect the statistics of coherent vortices to be universal. They should rather depend on the type of viscous dissipation and the Reynolds number (if the latter is not too large).

\section{Conclusion}

The evolution of strongly localized vortices (with a single sign of vorticity, without forcing and without dissipation) in a flow in the presence of stochastic perturbations has been analyzed. The analysis have been made in the framework of a 2-D point-vortex model.

It was shown that relaxation of system to an axially symmetrical configuration can be stoped due to the spontaneous formation of a regular structure of localized vortices. The observed clusters can be explained without an incorporating of special physics arguments beyond a 2-D euler model. A good quantitative agreement between plasma experiments at $R e \rightarrow \infty$ and the numerical integration of the proposed model in the evolution of the great number of vortices, was found.

An analytical consideration and a numerical analysis were performed to find out if the predictions of statistical mechanics (it predict a relaxation of system to the axially symmetrical configuration) could be due to the existence of a fluctuating part of the hamiltonian in the model and the absence of dissipation. It was be noted that even a small level of numerical dissipation can completely destroy the observed process of a vortex structure formation.

Edited by: A. Provenzale

Reviewed by: two referees 


\section{References}

Bartello, P. and Warn, T.: Self-similarity of decaying twodimensional turbulence, J. Fluid Mech., 326, 357-372, 1996.

Batchelor, G. K.: An Introduction to Fluid Dynamics, Cambridge University Press, p. 537, 1967.

Batchelor, G. K.: Computation of the energy spectrum in homogeneous two-dimensional turbulence, Phys. Fluids Suppl. II, 12, 233, 1969.

Basdevant, C., Legras, B., Sadourny, R., and Béland, M.: A study of barotropic model flows: intermittency, waves and predictability, J. Atmos. Sci., 38, 2305-2326, 1981.

Benzi, R., Patarnello, S., and Santangelo, P.: Self-similar coherent structures in two-dimensional decaying turbulence, J. Phys. A, 21, 1221-1237, 1988.

Benzi, R., Colella, M., Briscolini, M., and Santangelo, P.: A simple point vortex model for two-dimensional dcaying turbulence, Phys. Fluids A, 4, 1036-1939, 1992.

Brands, H., Chavanis, P. H., Pastmanter, R., and Sommeria, J.: Maximum entropy versus minimum enstrophy vortices, Phys. Fluids, 11(11), 3465, 1999.

Carnevale, G. F., McWilliams, J. C., Pomeau, Y., Weiss, J. B., and Young W. R.: Evolution of vortex statistics in two-dimensional turbulence, Phys. Rev. Lett., 66, 2735-2737, 1991.

Carnevale, G. F., McWilliams, J. C., Pomeau, Y., Weiss, J. B., and Young W. R.: Rates, pathways, and end states of non linear evolution in decaying two-dimensional turbulence: Scaling theory versus selective dacay, Phys. Fluids A, 4, 1314-1316, 1992.

Chasnov, J. R.: On the decay of two-dimensional homogeneous turbulence, Phys. Fluids, 9, 171-180, 1997.

Chasnov, J. R. and Herring, J. R.: Self-Similar Decay of twoDimensional Turbulence, Advances in turbulence, 415-418, 1998.

Chirikov, B. V.: Research cocerning the theory of nonlinear resonance and the stochasticity, Preprint 267, 1969, Novosibirsk, Institute of Nuclear Physics, Engl. Trans., CERN Trans., 71-40, 1971.

Chirikov, B. V.: Motion stability problem of a charged particle in a magnetic snare [Problema ustoiichivosti dvijenija zarjajennoii chastizy v magnitnoii lovushke.], Preprint 78-23, Budker Institute of Nuclear Physics, Novosibirsk, 1978.

Chirikov, B. V.: A universal instability of many-dimensional oscillator systems, Phys. Rep., 52(5), 263-379, 1979.

Danilov, S., Dolzhanskii, F. V., Dovzhenko, V. A., and Krymov, V. A.: Experiments on free decay of quasi-two-dimensional turbulent flows, Phys. Rev. E, 65, 036316, 2002.

Danilov, S. D. and Gurari, D.: Quasi-two-dimensional turbulence. Physics - Uspekhi, 43(9), 863-900, 2000.

Dritschel, D. G.: vortex properties of two-dimensional turbulence, Phys. Fluids A, 5, 984-997, 1993.

Fine, K. S., Cass, A. S., Flynn, W.-G., and Driscoll, C.-F.: Relaxation of 2-D turbulence to vortex crystals, Phys. Rev. Lett., 75, 3277, 1995.

Fornberg, B. J.: A numerical study of 2-D turbulence, Comput. Phys., 25, 1-37, 1977.

Goncharov, V., Pavlov, V. : Multipetal Vortex Structures in Two-Dimensional Models of Geophysical Fluid Dynamics and Plasma, J. Experimental and Theoretical Phys., 92, 4, 594-607 2001.

Holloway, G.: Statistical geofluid mechanics, Ann. Rev. Fluid Mech., 18, 91-147, 1986.

Huang, X. P. and Driscoll, C. F.: Relaxation of 2-D turbulence to a mataequilibrium near the minimum enstrophy state, Phys. Rev. Lett. 72, 2187, 1994.

Kapitsa, P. L.: Dynamic stability of a pendulum with oscillating suspension point, JETP, 21(5), 588-597, 1951.

Landau, L. D. and Lifchitz, E.-M.: Physique statistique, vol. 5, Nauka, Moscou, 1979a.

Landau, L. D. and Lifchitz, E.-M.: Cinétique physique, vol. 10, Mir, Moscou, p. 26, 1979 b.

Landau, L. D. and Lifshitz, E. M.: Fluid Mechanics, 2nd ed., rev., Pergamon Press, Oxford, New York, 1987.

Legras, B. and Dritschel, D. J.: A comparison of the contour surgery and pseudospectral method, Comput. Phys., 104, 287, 1993.

Matthaeus, W. H., Stribling, W. T., Martinez, D., Oughton, S., and Montgomery, D.: Selective decay and coherent vortices in two-dimensional incompressible turbulence, Phys. Rev. Lett., 66, 2731-2734, 1991.

Matthaeus, W. H., Stribling, W. T., Martinez, D., Oughton, S., and Montgomery D.: Decaying, two-dimensional, Navier-Stockes turbulence at very long times, Physica D, 51, 531-538, 1991.

Mariotti, A., Legras, B., and Dritschel, D. G.: Vortex stripping erosion of coherent structures in two-dimensional flows, Phys. Fluids, 6, 3954-3962, 1994.

Marteau, D., Cardoso, O., and Tabeling, P.: Equilibrium states of two-dimensional turbulence: an experimental study, Phys. Rev. E, 51(5), 5124, 1995.

McWilliams, J. C.: The emergence of isolated coherent vortices in turbulent flow, J. Fluid Mech., 146, 21, 1984.

McWilliams, J. C.: The vortices of geostrophic turbulence, J. Fluid Mech., 219, 387-404, 1990a.

McWilliams, J. C.: A demonstration of the suppression of turbulent cascades by coherent vortices in two-dimensional turbulence, Phys. Fluids A, 2, 547-552, 1990b.

Migdal, A. B.: Qualitative methods in quantum theorie, Nauka, 1975.

Miller, J., Weichman, P. B., and Cross, M. C.: Statistical mechanics, Euler's equations, and Jupiter's Red Spot, Phys. Rev. A , 45(4), 2328-2359, 1992.

Onsager, L.: Statistical hydrodynamics, Nuevo Cimento Suppl. 6, 279, 1949.

Pavlov, V., Buisine, D. and Goncharov, V. Formation of vortex clusters on a sphere, Nonlin. Processes Geophys., 8, 9-19, 2001.

Pavlov, V., Buisine, D., and Decossin, S.: Whether two-dimensional turbulence evolves to a unique state, Phys. Fluids, 14(11), 37393745, 2002.

Pointin, Y. B., and Lundgren, T.-S.: Statistical mechanics of twodimensional vortices in a bounded container, Phys. Fluids, 19, 1459, 1976.

Salmon, R.: Lectures on Geophysical Fluid Dynamics, Oxford University Press, 1998.

Santangelo, P., Benzi, R., and Legras, B.: The generation of vortices in high-resolution, two-dimnsional decaying turbulence and the influence of initial conditions on the breacking of self-similarity, Phys. Fluids A, 1, 1027-1034, 1989.

Sommeria, J., Staquet, C., and Robert, R.: Final equilibrium state of a two-dimensional shear layer, J. Fluid Mech., 233, 661, 1991.

Weiss, J. B. and McWilliams, J. C.: Temporal scaling behavior of decaying two-dimensional turbulence, Phys. Fluids A, 5, 608$621,1993$.

Zaslavskii, G. M. and Sagdeev, P. Z.: Introduction in Nonlinear Physics, M., Nauka, 1988. 